\title{
Two sides of the same coin: communication technology, media use, and our kids' health
}

\author{
Anna Ševčíková
}

Published online: 3 February 2015

(C) Swiss School of Public Health 2015

During the last several decades, there has been an explosion in the diversity of media available. TV, computers, the Internet, mobile phones, and most recently, smart phones have come to dominate the lives of many children and adolescents and the spaces where they spend their leisure time.

This special issue on "Communication Technology, Media Use, and Health of Our Kids" represents an attempt to understand what living and growing up with media means to them by disentangling selected associations between media use and measures of youngsters' health. It presents articles from all corners of the world, ranging from Taiwan, Australia, Brazil, Canada, the USA, and Israel to many European countries. A substantial proportion of the studies are the result of large-scale cross-sectional or longitudinal surveys.

Health can be viewed from the perspective of wellbeing, a multidimensional construct according to which quality of life is framed in terms of physical, emotional, mental, social, and behavioral components (Janse et al. 2004). Media use is a two-sided coin that may affect both physical and psychological health. The present compilation of research shows that it is necessary to consider media's impact in both spheres to come up with practices that moderate the extremes of media use and maximize the benefits, while minimizing the harm. Furthermore, this special issue takes into account the fact that the young

This editorial is part of the special issue "Communication Technology, Media Use and the Health of Our Kids".

\section{A. Ševčíková ( $\square)$}

Faculty of Social Studies, Institute for Research on Children, Youth and Family, Masaryk University, Joštova 10, 60200 Brno, Czech Republic

e-mail: asevciko@fss.muni.cz population is no longer exposed only to one traditional medium, i.e., TV. The boom in electronic devices has expanded the number of attractive screen-based activities, which brings new challenges in how to monitor total screen time and disentangle the potential effects of each device on physical functioning.

Prior studies have clearly documented that communication technology can be misused for bullying, and some of them suggest traditional or school bullying may move into virtual worlds (Hinduja and Patchin 2013). Despite an increasing body of research work, there is still ongoing debate on how cyberbullying relates to traditional bullying, how cyberbullying impacts children, and what risk factors are linked to cyber-victimisation and cyberbullying behavior. With five articles relating to these themes, this special issue shows the extent to which the risk factors for involvement in cyberbullying are complex and range from interactions with traditional bullying scenarios and underlying psychological problems. The interplay of the factors may affect patterns of internet use and, according Hemphill et al. (2015), even result in specific negative outcomes depending on perpetration/victimisation status.

The content of the special issue also emphasizes the importance of focusing on specific activities and drawing distinctions between them. This approach, which goes beyond conceptualizing computer, internet or mobile phone use as a general single behavior, allows researchers to discern which of the broad scale of internet/PC activities may support or inhibit young people's development. This methodology is particularly warranted when we want to avoid generalizing the negative effects of some behaviors to media/technology use as a whole, and to disentangle the complex associations between media use and health. Then, as in the example of the studies by Boniel-Nissim et al. 
(2014) and Gommans et al. (2014), it is possible to observe how one activity in a specific context and under specific conditions may act as either a risk factor or a protective factor.

Similarly, descending from a general focus on games to the level of game types or features may be a promising approach for studying health in connection with online/ computer games, one of the most popular activities among children and adolescents (Livingstone et al. 2011). The articles selected for the special issue corroborate the existing body of research in showing an association with other risk factors. For instance, Chang et al. (2014) and Rasmussen et al. (2014) found a link between game playing and bullying (both perpetration and victimization). Further analysis of the role of game type seems necessary for elucidating this association. Among the more severe risks associated with gaming is Internet Gaming Disorder, which is listed as a future research need in the DSM-5 (American Psychiatric Association 2013), and the association between virtual world-based games and excessive internet use reported Blinka et al. (2014) indicates that some players of these games might be prone to developing symptoms of this disorder.

Last but not least, this special issue represents an overview of how the younger generation has been using media and communication technology throughout the past decade. Media use is evidently still evolving as an increasing number of online platforms and networked devices become available. The question of what our kids do in their (media saturated) bedrooms needs to be reformulated to what happens in their pockets, as media are accessible anytime and anywhere. One fact is certain: young people will remain the most enthusiastic media users, although media usage is limited by the social, cultural, and physical context as well as by the number of hours in a day.

\section{References}

American Psychiatric Association (2013) Fifth edition of statistical and mental disorders. American Psychiatric Publishing, Arlington

Blinka L, Škařupová K, Ševčíková A, Wölfling K, Müller KW, Dreier M (2014) Excessive internet use in European adolescents: what determines differences in severity? Int J Public Health. doi:10. 1007/s00038-014-0635-x

Boniel-Nissim M, Tabak I, Mazur J, Borraccino A, Brooks F, Gommans R, van der Sluijs W, Zsiros E, Craig W, Harel-Fisch Y, Finne E (2014) Supportive communication with parents moderates the negative effects of electronic media use on life satisfaction during adolescence. Int J Public Health. doi:10.1007/ s00038-014-0636-9

Chang FC, Chiu CH, Miao NF, Chen PH, Lee CM, Huang TF, Pan YC (2014) Online gaming and risks predict cyberbullying perpetration and victimization in adolescents. Int $\mathrm{J}$ Public Health. doi:10.1007/s00038-014-0643-x

Gommans R, Stevens GW, Finne E, Cillessen AH, Boniel-Nissim M, ter Bogt TF (2014) Frequent electronic media communication with friends is associated with higher adolescent substance use. Int J Public Health. doi:10.1007/s00038-014-0624-0

Hemphill S, Kotevski A, Heerde J (2015) Longitudinal associations between cyber-bullying perpetration and victimization and problem behavior and mental health problems in young Australians. Int J Public Health. doi:10.1007/s00038-014-0644-9

Hinduja S, Patchin JW (2013) Social influences on cyberbullying behaviors among middle and high school students. J Youth Adolesc 42:711-722

Janse AJ, Gemke RJ, Uiterwaal CS, Van Der Tweel I, Kimpen JL, Sinnema G (2004) Quality of life: patients and doctors don't always agree: a meta-analysis. J Clin Epidemiol 57:653-661

Livingstone S, Haddon L, Görzig A, Ólafsson K (2011) Risks and safety on the internet: the perspective of European children. Full findings. LSE, London: EU Kids Online. Retrieved October 14, 2013, from http://www.lse.ac.uk/media@1se/research/EUKids Online/EU\%20Kids\%20II\%20(2009-11)/EUKidsOnlineIIReports/ D4FullFindings.pdf

Rasmussen M, Meilstrup CR, Bendtsen P, Pedersen TP, Nielsen L, Madsen KR, Holstein BE (2014) Perceived problems with computer gaming and Internet use are associated with poorer social relations in adolescence. Int J Public Health. doi:10.1007/ s00038-014-0633-Z 